\title{
Keratosis pilaris atrophicans faciei: An observational, descriptive, retrospective clinical study
}

\author{
GYULA LÁSZLÓ FEKETE ${ }^{1}$, LÁSZLÓ FEKETE ${ }^{2}$, NICOLETA NEAGU ${ }^{2}$, \\ VLADIMIR BACÂREA ${ }^{3}$, MIRUNA DRĂGĂNESCU ${ }^{4}$ and ILARIE BRIHAN ${ }^{5}$
}

\begin{abstract}
${ }^{1}$ Department of Dermatology, Dermatology Clinic, 'George Emil Palade' University of Medicine, Pharmacy, Science and Technology, 540139 Târgu Mureș; ${ }^{2}$ Department of Dermatology, Mureș County Hospital, 540015 Târgu Mures; ${ }^{3}$ Department of Medical Research Methodology, 'George Emil Palade’ University of Medicine, Pharmacy, Science and Technology, 540139 Târgu Mureș; ${ }^{4}$ Faculty of Medicine and Pharmacy, Medical Department, 'Dunărea de Jos' University, 800010 Galati; ${ }^{5}$ Department of Dermatology, Dermatology Clinic, Faculty of Medicine and Pharmacy, University of Oradea, 410073 Oradea, Romania
\end{abstract}

Received July 6, 2021; Accepted August 5, 2021

DOI: $10.3892 / \mathrm{etm} .2021 .10766$

\begin{abstract}
Keratosis pilaris atrophicans faciei (KPAF) is a hereditary follicular disorder, an atrophicans subtype of keratosis pilaris (KP) with a highly elusive diagnosis. Clinically, it presents with follicular, horny papules surrounded by an erythematous halo of the cheeks, forehead, chin and eyebrows, and it is followed by a gradual hair loss on the lateral margins of the eyebrows. The onset is as early as a few months after birth, but it is mainly diagnosed in children and adolescents and it can persist through adulthood. At present, the natural progression of the disease is poorly understood, which makes a correct diagnosis highly unlikely. The aim of the present study was to describe the clinical characteristics of KPAF in patients encountered in daily practice, in order to find common characteristics that may aid in the earlier recognition of the disease. An observational, descriptive, retrospective study was performed on 14 patients diagnosed with KPAF between January 2000 and December 2020. The mean age at diagnosis was 17.04 years and the onset of clinical symptoms appeared at a mean age of 4.85 years. The first clinical symptom was
\end{abstract}

Correspondence to: Dr László Fekete, Department of Dermatology, Mureș County Hospital, 12 Gheorghe Doja Street, 540015 Târgu Mures, Romania

E-mail: dermafek@yahoo.com

Dr Miruna Drăgănescu, Faculty of Medicine and Pharmacy, Medical Department, 'Dunărea de Jos' University, 35 Al. I. Cuza Street, 800010 Galati, Romania

E-mail:1acifek@yahoo.com

Abbreviations: KPAF, keratosis pilaris atrophicans faciei; $\mathrm{KP}$, keratosis pilaris

Key words: keratosis pilaris atrophicans faciei, keratosis pilaris, eyebrow loss, erythema of the face
KP involving either the upper or lower limbs, or both. Then, erythema of the face appeared at a mean age of 7.21 years, keratotic papules on the face at a mean age of 8.35 years and, finally, loss of hair on the lateral margins of the eyebrows at a mean age of 14 years. The patients also had concomitant xerosis cutis, multiple mole syndrome, acne, contact dermatitis and Laugier-Hunziker syndrome. Evidence of disease progression, associations, as well as efficacious treatment measures are lacking. An earlier diagnosis potentially allows for a more efficacious, targeted treatment option. Either topical emollients, systemic retinoids or laser therapy may prove effective for each patient individually.

\section{Introduction}

Keratosis pilaris (KP) is a common skin disorder that can also be associated with less common variants and rare subtypes, including keratosis pilaris rubra, erythromelanosis follicularis faciei et colli and the spectrum of keratosis pilaris atrophicans (1). The atrophicans subtypes, which are even more uncommon, include keratosis pilaris atrophicans faciei (KPAF), atrophoderma vermiculatum, and keratosis follicularis spinulosa decalvans (1). KPAF, also known as ulerythema ophryogenes, is a hereditary disorder characterized by altered follicular keratinization and inflammation, which leads to subsequent atrophy (2). Clinically, it presents with follicular, horny papules surrounded by an erythematous halo of the cheeks, forehead, chin and eyebrows, and it is followed by a gradual loss of hair. These cutaneous manifestations appear sequentially: Erythematous follicular papules of the face are usually the first sign, followed by a gradual hair loss on the lateral part of the eyebrows and finally follicular scar-like follicular atrophy (3). KPAF occurs sporadically; however, autosomal dominant and autosomal recessive inheritance have been described (3). In some cases, an autosomal recessive mutation in the desmoglein 4 (DSG4) gene, as well as the LDL receptor related protein 1 (LRPI) gene, have been involved $(4,5)$. The onset is as early as a few months after birth, with erythema and 
keratotic follicular papules affecting the lateral third of the eyebrows (6); however, KPAF is mainly diagnosed in children and adolescents, and it can persist through adulthood. Most of the subjects have concomitant KP of the extremities and trunk. Given the clinical variability of the particular cutaneous signs, insufficient diagnosis and misdiagnosis are frequent. In the majority of cases, the first clinical signs appeared on the face and are attributed to other diseases, thus making the real incidence of the disease unknown (7).

KPAF is a rare hereditary disorder with well-defined clinical features, but with variable evolution. At present, the natural progression of the disease is poorly understood, which makes a correct diagnosis highly unlikely. Thus, the aim of this observational, descriptive, retrospective study was to describe the clinical characteristics of KPAF in patients encountered in daily practice, in order to find common characteristics that may aid in the earlier recognition of the disease.

\section{Patients and methods}

Cases. To identify common characteristics that may aid in the earlier recognition of KPAF, an observational, descriptive, retrospective clinical case series study on 14 patients with KPAF was performed at the Dermatology Clinic of Târgu-Mures, Romania, between January 2000 and December 2020. In this 20-year period, 14 out of 76,440 patients were diagnosed with KPAF. Mean age at diagnosis was 17.04 years, with the youngest patient being diagnosed at the age of 3 years and the eldest at the age of 22 years. The sex ratio was $\mathrm{M} / \mathrm{F}=4 / 1$. The diagnosis was established through clinical examination. Patients were residents of the urban area and were members of wealthy families.

One investigator evaluated the patients and collected data and another investigator, who was blinded to clinical cases and treatment choices, performed outcome assessment. Data acquisition was performed by clinical examination and telephone interview. The patients were followed-up for four years after KPAF diagnosis. Limitations of the current study lie in the relatively low number of patients and in confirmation bias in reporting, since clinical assessment, diagnosis and treatment were performed by a single investigator. Mild form of facial erythema was defined as few papules and mild erythema, whereas severe form was defined as predominant papules and severe erythema.

\section{Results}

In this 20 -year period, 14 out of 76,440 patients were diagnosed with KPAF. Mean age at diagnosis was 17.04 years, with the youngest patient being diagnosed at the age of 3 years and the eldest at the age of 22 years. The sex ratio was $\mathrm{M} / \mathrm{F}=4 / 1$. The onset of clinical symptoms appeared at a mean age of 4.85 years (2-12 years) and the time between the appearance of the first clinical symptoms to KPAF diagnosis was 9.21 years (1-16 years).

The majority of the 14 patients $(71.43 \%)$ presented with keratosis pilaris (KP) involving only the upper limb as the first clinical sign; the remaining $28.57 \%$ had both upper and lower limb involvement. The second clinical manifestation, erythema of the face, appeared at a mean age of 7.21 years (2-16 years),
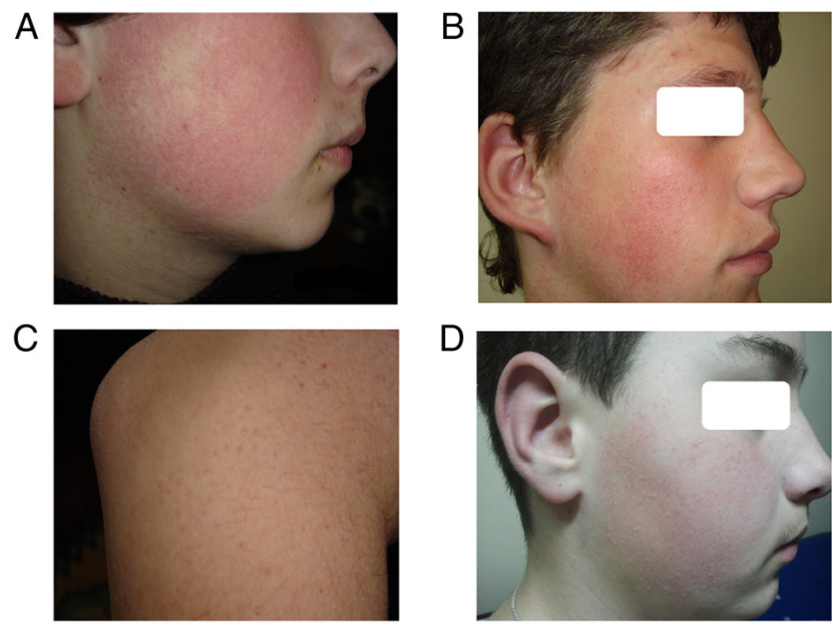

Figure 1. Clinical aspects of KPAF. (A) Follicular, horny papules surrounded by an erythematous halo, on a large surface of the cheeks. (B and D) Follicular, horny papules surrounded by an erythematous halo on the cheeks; partial hair loss in the lateral margins of the eyebrows. (C) Erythematous follicular, horny papules involving the lower extremities.

after a mean period of 3.66 years (1-7 years) since the onset of $\mathrm{KP}$ of the extremities, in 9 out of 14 patients; for the remaining 5 patients, both KP and facial erythema appeared concomitantly. Of the 14 patients, 7 patients had mild forms and 7 had severe forms. The third clinical manifestation, keratotic papules on the face, appeared at a mean age of 8.35 years (3-14 years), after a mean period of 1.75 years (1-5 years) after facial erythema and 3.69 years (1-8 years) after KP of the extremities, in 13 out of 14 cases. In one case, though, keratotic papules on the face appeared concomitantly with KP and preceded the appearance of facial erythema. In addition, 8 patients presented skin atrophy. Loss of hair in the lateral margins of the eyebrows appeared at a mean age of 14 years (3-22 years), after a mean period of 9.14 years (1-16 years) since the onset of the first symptom, KP of the extremities, 6.78 years (1-16 years) after facial erythema and 6.07 years (1-15 years) after keratotic papules on the face, for 13 out of 14 patients. For one of the patients, loss of hair in the lateral margins of the eyebrows was concomitant with the appearance of keratotic papules on the face and these symptoms appeared one year after the onset of $\mathrm{KP}$ and facial erythema; this case was diagnosed as KPAF at the age of 3 years (Fig. 1). In all of these cases, KPAF was not the first diagnosis. Habitual erythema, keratosis pilaris, contact dermatitis, comedonal acne, infantile acne and atopic dermatitis were the misdiagnoses. Furthermore, $78.57 \%$ of the patients had associated xerosis cutis, $35.71 \%$ also had multiple mole syndrome, $14.28 \%$ had acne, $7.14 \%$ had contact dermatitis and 7.14\% had Laugier-Hunziker syndrome (Table I). Topical treatments were recommended: emollients, keratolytics (containing lactic acid, salicylic acid, or urea), benzoyl peroxide, with mild, temporary improvement.

\section{Discussion}

Keratosis pilaris atrophicans faciei (KPAF) is an early-onset disease with an elusive diagnosis, due to the long-term 


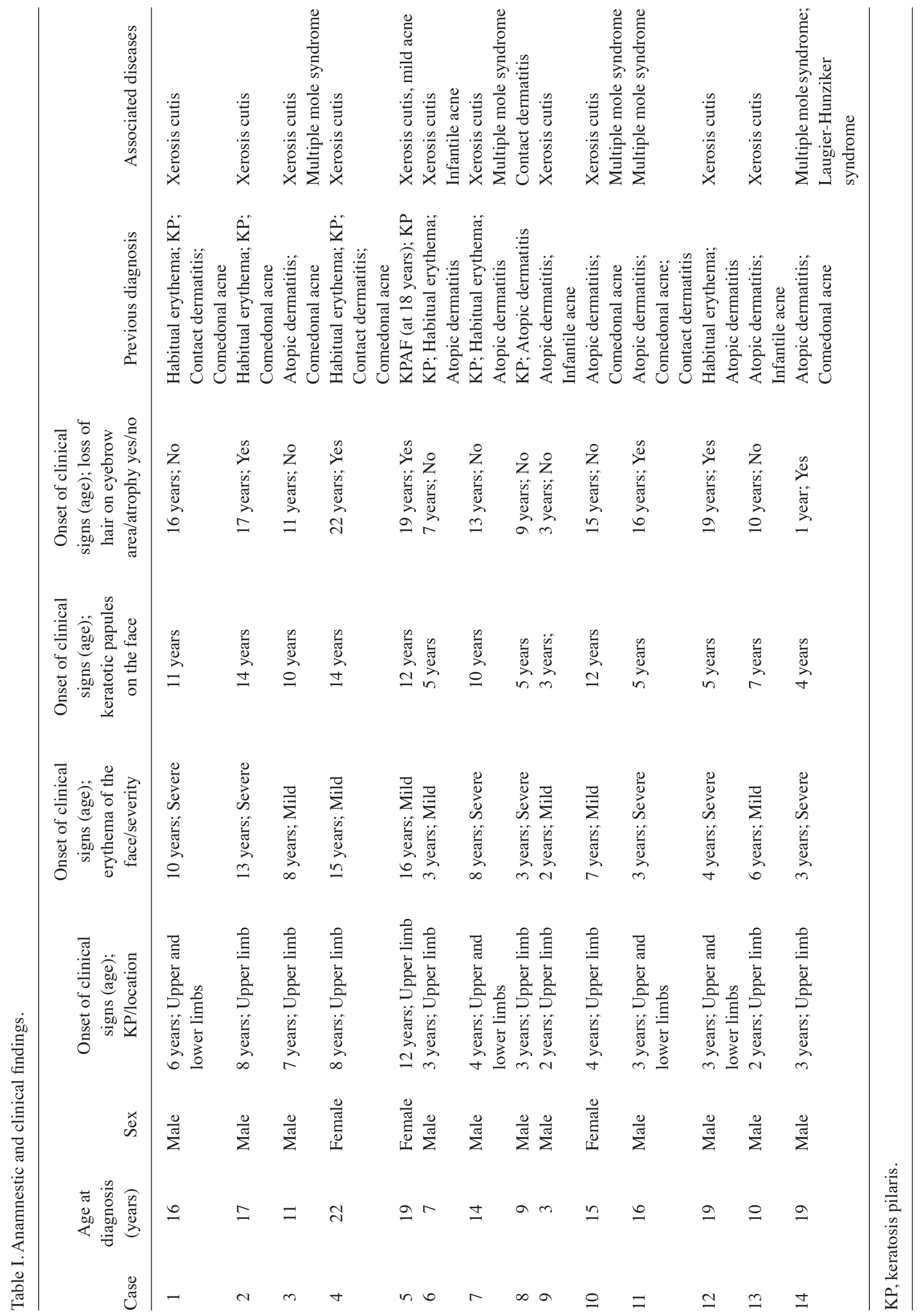


progression of the symptoms. Results of the present study revealed that from the appearance of the first symptom to a correct diagnosis 9.21 years had passed, with a mean age at diagnosis of 17.04 years. Considering that the first clinical signs appeared as early as the age of 1 year, misdiagnosis delayed a correct assessment of the clinical image. The majority of the cases $(64.28 \%)$ had a sequential progression; first, they presented KP involving the limbs, then, after a mean of 3.66 years facial erythema appeared; the third symptom, keratotic papules on the face, occurred after a mean of 1.75 years and 6.07 years later loss of hair appeared on the lateral margin of the eyebrows. Patients included in the present study also had associated xerosis cutis, multiple mole syndrome, acne, contact dermatitis and Laugier-Hunziker syndrome. In the literature, more severe afflictions have been described in association with KPAF including Noonan syndrome (8), Zouboulis syndrome (2), Cornelia de la Lange syndrome (6), Rubinstein-Taybi syndrome (9), and woolly hair syndrome (10). Furthermore, Wang and Orlow summarized KP associations with various neuro-cardio-facial-cutaneous syndromes, ectodermal dysplasias, and neuro developmental disorders, as well as KP-induced drug reactions (11). Differential diagnosis includes a large spectrum of diseases that affect children and adolescents, and which progresses with facial erythema, from habitual erythema, rosacea, atopic dermatitis, comedonal acne, to KP subtypes, including keratosis pilaris rubra faciei, erythromelanosis follicularis faciei et colli, atrophoderma vermiculatum, Darier-White disease, and pityriasis folliculorum (12-14).

An early diagnosis allows for a targeted treatment option. The patients in the current study were recommended to receive emollients, benzoyl peroxide and keratolytics containing lactic acid, salicylic acid, or urea, with only mild temporary improvement. Other therapies have been described, with encouraging results including pulsed dye laser (PDL) (15) and intense pulsed laser (16). Additionally, Apalla et al described a case of atrophoderma vermiculatum which responded to systemic isotretinoin (17). Furthermore, Wang et al summarized various topical treatments with reasonably adequate effect in patients with KP. Thiese included lactic acid, salicylic acid, retinoids, aquaphor, fractional prickle coral calcium, spray-on nitrosomonas eutropha mist, chlorine dioxide complex cleanser, as well as various lasers: PDL, 532-nm potassium titanyl phosphate laser (KTPL), alexandrite laser, long-pulsed diode laser, Q-switched Nd:YAG laser, and fractional carbon dioxide laser $(11,18,19)$. There are no available treatments to prevent or reduce the atrophy in $\mathrm{KPAF}$.

Limitations of the current study lie in the relatively low number of patients and in confirmation bias in reporting, since clinical assessment, diagnosis and treatment were performed by a single investigator.

In summary, KP is a very common skin condition, often dismissed as a cosmetic matter, which frequently leads to missed diagnoses of associated diseases, hereditary syndromes or even adverse events of certain medications. Evidence of disease progression, associations, as well as efficacious treatment measures is lacking. Further case series investigating the chronology of symptoms, as well as the efficacy, tolerability and recurrence rates are necessary in deciphering KPAF.

\section{Acknowledgements}

Not applicable.

\section{Funding}

No funding was received.

\section{Availability of data and materials}

All data generated or analyzed during this study are included in this published article.

\section{Authors' contributions}

GLF was responsible for the clinical management of the cases, the evaluation and analysis of data, and writing of the manuscript. LF was responsible for corrections and preparation of the manuscript. NN, MD and BV were responsible for data search and revision of the manuscript. IB contributed to writing the manuscript. The final version of the article has been read and approved by all authors. GLF and NN are responsible for confirming the authenticity of the raw data.

\section{Ethics approval and consent to participate}

Written informed consent from the patients was obtained.

\section{Patient consent for publication}

Written informed consent from the patients was obtained.

\section{Competing interests}

The authors declare that they have no competing interests.

\section{Authors' information}

GLF is an Associate Professor of Dermatology, Dermatology Department, Dermatology Clinic, 'George Emil Palade' University of Medicine, Pharmacy, Science and Technology, Târgu Mureş, Romania.

\section{References}

1. Egan O, Luther EM and Oakley A: Keratosis pilaris atrophicans. Edited Gus Mitchel, 2020 https://dermnetnz.org/topics/keratosis-pilaris-atrophicans-faciei/.

2. Liakou AI, Esteves de Carvalho AV and Nazarenko LP: Trias of keratosis pilaris, ulerythema ophryogenes and 18p monosomy: Zouboulis syndrome. J Dermatol 41: 371-376, 2014.

3. Callaway SR and Lesher JL Jr: Keratosis pilaris atrophicans: Case series and review. Pediatr Dermatol 21: 14-17, 2004.

4. Cohen-Barak E, Danial-Farran N, Hammad H, Aleme O, Krauz J, Gavishi E, Khayat M, Zivi M and Shalev S: Desmoglein 4 mutation underlies autosomal recessive keratosis pilaris atrophicans. Acta Derm Venereol 98: 809-810, 2018.

5. Klar J, Schuster J, Khan TN, Jameel M, Mäbert K and Forsberg L: Whole exome sequencing identifies LRP1 as a pathogenic gene in autosomal recessive keratosis pilaris atrophicans. J Med Genet 52: 599-606, 2015.

6. Flórez A, Fernández-Redondo V and Toribio J: Ulerythema ophryogenes in Cornelia de Lange syndrome. Pediatr Dermatol 19: 42-45, 2002. 
7. Morton CM, Bhate C, Janniger CK and Schwartz RA: Ulerythema ophryogenes: Updates and insights. Cutis 93: 83-87, 2014.

8. Li K, Ann Thomas M and Haber RM: Ulerythema ophryogenes, a rarely reported cutaneous manifestation of Noonan syndrome: Case report and review of the literature. J Cutan Med Surg 17: 212-218, 2013

9. Gómez Centeno P, Rosón E, Peteiro C, Mercedes Pereiro M and Toribio J: Rubinstein-Taybi syndrome and ulerythema ophryogenes in a 9-year-old boy. Pediatr Dermatol 16: 134-136, 1999.

10. Chien AJ, Valentine MC and Sybert VP: Hereditary woolly hair and keratosis pilaris. J Am Acad Dermatol 54 (Suppl 2): S35-S39, 2006.

11. Wang JF and Orlow SJ: Keratosis pilaris and its subtypes: Associations, new molecular and pharmacologic etiologies, and therapeutic options. Am J Clin Dermatol 19: 733-757, 2018.

12. Fekete GL, Boda D, Căruntu C and Fekete L: Paraneoplastic pityriasis rubra pilaris in association with prostate carcinoma: A case report and literature review. Exp Ther Med 18: 5052-5055, 2019.

13. Kanakpur SH and Caculo DU: Rare ocular manifestations in keratosis follicularis (Darier-White disease). Indian J Ophthalmol 65: 874-876, 2017
14. Tatu AL and Violeta VC: Pityriasis folliculorum of the back thoracic area: Pityrosporum, keratin plugs, or Demodex involved? J Cutan Med Surg 21: 441-447, 2017.

15. Alcántara González J, Boixeda P, Truchuelo Díez MT and Fleta Asín B: Keratosis pilaris rubra and keratosis pilaris atrophicans faciei treated with pulsed dye laser: Report of 10 cases. J Eur Acad Dermatol Venereol 25: 710-714, 2010.

16. Rodríguez-Lojo R, Pozo JD, Barja JM, Pineyro F and Perez-Varela L: Keratosis pilaris atrophicans: Treatment with intense pulsed light in four patients. J Cosmet Laser Ther 12: 188-190, 2010.

17. Apalla Z, Karakatsanis G, Papageorgiou M, Kastoridou C and Chademenos G: A case of atrophoderma vermiculatum responding to systemic isotretinoin. J Dermatol Case Rep 3: 62-63, 2009.

18. Bacârea PF, Neda I, Daniliuc CG, Bacârea A and Silaghi DL: Chiral selectivity in the basic or acid $\alpha$-amino acids homomeric $\mathrm{Cu}(\mathrm{II})$ complexes range. Rev Chim 63: 489-498, 2012.

19. Ianosi SL, Batani A, Ilie MA, Tampa M, Georgescu SR, Zurac S, Boda D, Ianosi NG, Neagoe D, Calina D, et al: Non-invasive imaging techniques for the in vivo diagnosis of Bowen's disease: Three case reports. Oncol Lett 17: 4094-4101, 2019. 